\title{
PREFACE
}

\section{Chest Wall Reconstruction}

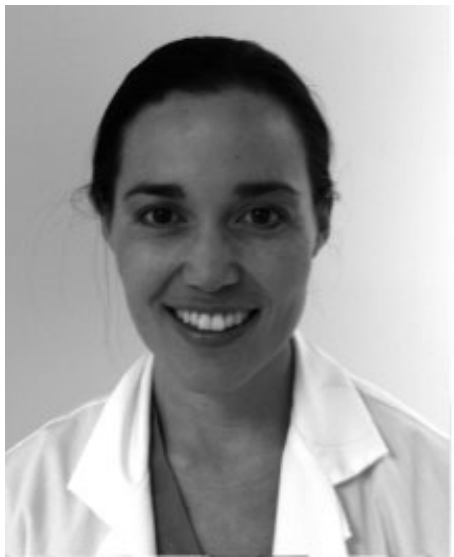

Karen K. Evans, M.D.

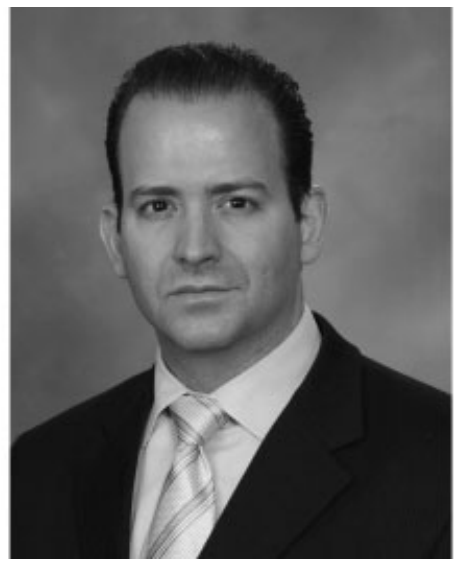

Samir Mardini, M.D.

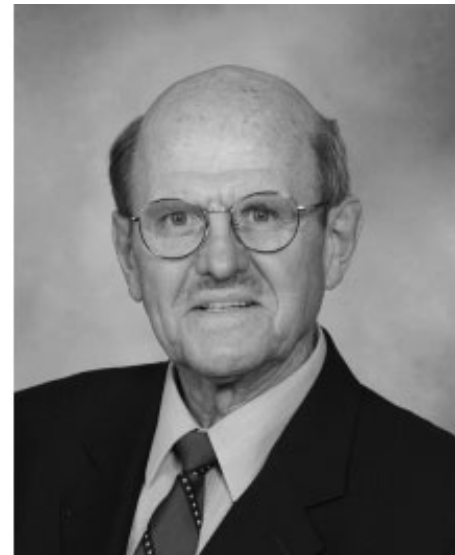

Phillip G. Arnold, M.D.
$W_{\text {e are excited to present this issue of Seminars }}$ in Plastic Surgery on "Chest Wall Reconstruction." This topic is multifaceted, multidisciplinary, and quite common. From specialized tertiary care academic centers to community practice, many plastic surgeons are faced with these patients. The principles involved in chest wall reconstruction reflect the tenets of reconstructive surgery: establish the diagnosis, optimize the patient, replace like with like, obliterate dead space, have a good backup plan, and follow the patient long term. In addition, the flaps involved in these cases are some of the most tried and true, historically relevant, and reliable flaps in our armamentarium.

For this issue, we asked the experts to write on all aspects of chest reconstruction and are confident that this is a complete resource on chest wall reconstruction. The background is set with an article on anatomy by Dr. Mark W. Clemens and colleagues and an extensive review of chest wall tumors by Dr. Blair Marshall and colleague Dr. Elizabeth A. David. The next article is a comprehensive and evidence-based analysis of sternal wound management, including timing of closure and use of antibiotics, by Dr. Erica Anderson and colleagues. Reconstruction articles begin with the article by Dr. Charles Butler and Dr. Raman Chaos Mahabir, who write extensively on their experience with bony reconstruction of the chest wall including mesh and biologic substitutes. The next two articles are the flap articles, from workhorse flaps to "plan B" flaps, and were respectively written by Drs. Arnold and Mardini and colleagues and by Dr. Joseph J. Disa and Dr. Evan Matros. Dr. Michael Sauerbier and colleagues then share their experience with the use of microsurgical free flaps for chest wall reconstruction. Importantly, the article by Dr. Hung-chi Chen and colleagues contributes to our understanding of intrathoracic pathology. Many times, the back is a forgotten anatomic unit of the chest wall; Dr. Hallock's comprehensive article on posterior trunk defects highlights flap reconstruction from the rudimentary to the most complex.

The final articles are equally as important. We cannot operate without a thorough knowledge of complications, and the perspective of Dr. David T. Netscher and colleagues on how to handle these difficult problems should be emphasized. The late complications that can occur after sternal reconstruction can be very troublesome and require innovative ways to overcome significant pain and disability. We are grateful for the perspective of Dr. Christopher J. Salgado and colleagues on this topic. Finally, as in many aspects of plastic
${ }^{1}$ Division of Plastic Surgery, Washington DC Veterans Affairs Medical Center, and Department of Plastic Surgery, Georgetown University Medical Center, Washington, DC; ${ }^{2}$ Department of Plastic Surgery, Mayo Clinic, Rochester, Minnesota.

Address for correspondence and reprint requests: Karen K. Evans, M.D., Division of Plastic Surgery, Washington DC Veterans Affairs Medical Center, 50 Irving Street, Washington, DC 20422 (e-mail: karenkim90@hotmail.com).
Chest Wall Reconstruction; Guest Editors, Karen K. Evans, M.D., Samir Mardini, M.D., and Phillip G. Arnold, M.D.

Semin Plast Surg 2011;25:3-4. Copyright (C) 2011 by Thieme Medical Publishers, Inc., 333 Seventh Avenue, New York, NY 10001, USA. Tel: +1(212) 584-4662.

DOI: http://dx.doi.org/10.1055/s-0031-1275165.

ISSN 1535-2188. 
surgery, the knowledge base of our colleagues in other specialties, such as pediatric thoracic surgery, is crucial to an understanding of how to combine our skills to treat patients. Dr. Anthony D. Sandler and colleagues show clearly how to treat congenital thoracic pathology.

We are grateful to our colleagues and dear friends in thoracic and cardiac surgery who have provided us the opportunity to see their patients. It is the collaboration with them and their graciousness in sharing their wisdom and experience that created our enthusiasm and dedication to this field.
It has been a pleasure to assimilate our friends and colleagues to organize what we hope the reader will find to be a comprehensive resource on this topic. We are grateful to all of the staff at Seminars in Plastic Surgery for this opportunity and thank them for all their help.

Karen K. Evans, M.D. ${ }^{1}$

Samir Mardini, M.D. ${ }^{2}$ Phillip G. Arnold, M.D. ${ }^{2}$

Guest Editors 